\title{
New evidence in hypertension and hyperlipidaemia
}

\section{N Chapman}

Heart 2004;90(Suppl IV):iv14-iv17. doi: 10.1136/hrt.2004.037556

The benefits of blood pressure lowering on the risk of cardiovascular disease are seen with all commonly used classes of antihypertensive drugs. Although different drug classes may have different effects on cause specific outcomes (such as myocardial infarction or stroke), no differences are observed between their overall effects on combined major cardiovascular end points. The choice of first line antihypertensive drug is therefore likely to be of less importance than the use of any effective drug, particularly since the majority of patients will need two or more agents to achieve blood pressure goals. Recent trials have provided good evidence that lowering cholesterol with statins reduces the risk of cardiovascular events in "high risk" patients, irrespective of initial cholesterol concentrations. The benefits of both blood pressure lowering and lipid lowering treatment appear to be proportional to the extent of the reduction of blood pressure and lipids achieved.

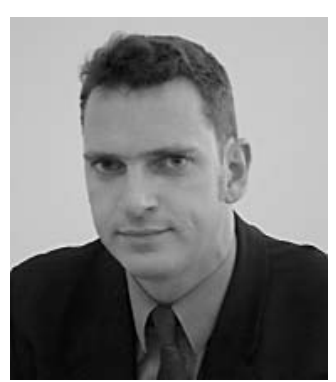

T he associations between blood pressure and the risks of stroke and coronary heart disease (CHD) are well established. There are strong, positive and continuous associations between blood pressure and risk, ${ }^{1}$ and there does not appear to be any lower level of blood pressure below which these associations fail to hold.

Meta-analyses (overviews) of early trials of blood pressure lowering, mainly with diuretics and $\beta$ blockers, clearly showed that lowering blood pressure reduces the risks of stroke and CHD. ${ }^{1}$ Recent overviews conducted by the blood pressure lowering treatment trialists' collaboration (BPLTTC) of placebo controlled trials have shown that both angiotensin converting enzyme (ACE) inhibitors and calcium channel blockers also reduce the risk of major cardiovascular events (a composite end point of CHD, stroke, heart failure or cardiovascular death) by about $20 \%$ (fig 1). ${ }^{2}$ In addition, an overview of trials that compared more intensive and less intensive blood pressure lowering strategies showed that more intensive strategies, while only achieving modestly greater reductions in blood pressure (mean 4/3 mm Hg), reduced the risk of major cardiovascular events by about 15\% (fig 1). ${ }^{2}$

Correspondence to: Dr Neil Chapman, St Mary's Hospital, London W2 INY, UK; neil.chapman@ st-marys.nhs.uk
In the past two years, several trials of the use of angiotensin receptor blockers (ARBs) in hypertensive patients have been published.

The losartan intervention for endpoint reduction in hypertension (LIFE) study involved 9193 patients aged 55-80 years with essential hypertension and left ventricular hypertrophy. ${ }^{3}$ Patients were randomised to losartan or atenolol, and followed up for a mean of 4.8 years. There were small differences between the blood pressure reductions achieved in the two groups. Compared with atenolol, losartan reduced the relative risk of the composite end point of death, myocardial infarction, or stroke by $13 \%$ $(p=0.02)$, primarily due to a reduction in the risk of stroke. There has been debate about the interpretation of this finding. One possibility is that losartan (or the ARBs as a class) has benefits beyond those of blood pressure lowering; alternatively, the observed difference may have been due to chance. A third possibility, however, is that atenolol was not an appropriate comparator-although diuretics and $\beta$ blockers were the most commonly used drugs in the early trials that demonstrated the benefits of blood pressure lowering on cardiovascular disease, ${ }^{1}$ the evidence of benefit is stronger for diuretics than for $\beta$ blockers.

The study on cognition and prognosis in the elderly (SCOPE) 4 involved 4964 participants with hypertension, aged 70-89 years, who were randomised to candesartan or placebo and followed up for a mean of 3.7 years. It was planned as a placebo controlled trial; however, the publication of other studies and updated guidelines during the patient recruitment period meant that, for ethical reasons, open label antihypertensive treatment was recommended in both groups for patients whose blood pressure remained uncontrolled. As a consequence, active antihypertensive treatment was widely used $(84 \%)$ in the control group and differences in blood pressure reduction between the two groups were modest $(3.2 / 1.6 \mathrm{~mm} \mathrm{Hg})$. There was no significant difference between the candesartan and control groups on the composite end point of major vascular events (cardiovascular death, non-fatal stroke, and non-fatal myocardial infarction).

The BPLTTC conducted an overview of trials that compared ARBs with other regimens (placebo or other active treatment). ${ }^{2}$ This

Abbreviations: $A C E$, angiotensin converting enzyme; ALLHAT, antihypertensive and lipid-lowering treatment to prevent heart attack trial; ARB, angiotensin receptor blocker; ASCOT-LLA, Anglo-Scandinavian cardiac outcomes trial-lipid lowering arm; BPLTTC, blood pressure lowering treatment trialists' collaboration; CHD, coronary heart disease; HPS, heart protection study; IDNT, irbesartan diabetic nephropathy trial; LDL, low density lipoprotein; LIFE, losartan intervention for endpoint reduction in hypertension; PROSPER, pravastatin in elderly individuals at risk of vascular disease; RENAAL, reduction of end points in NIDDM with the angiotensin II antagonist losartan; SCOPE, study on cognition and prognosis in the elderly 


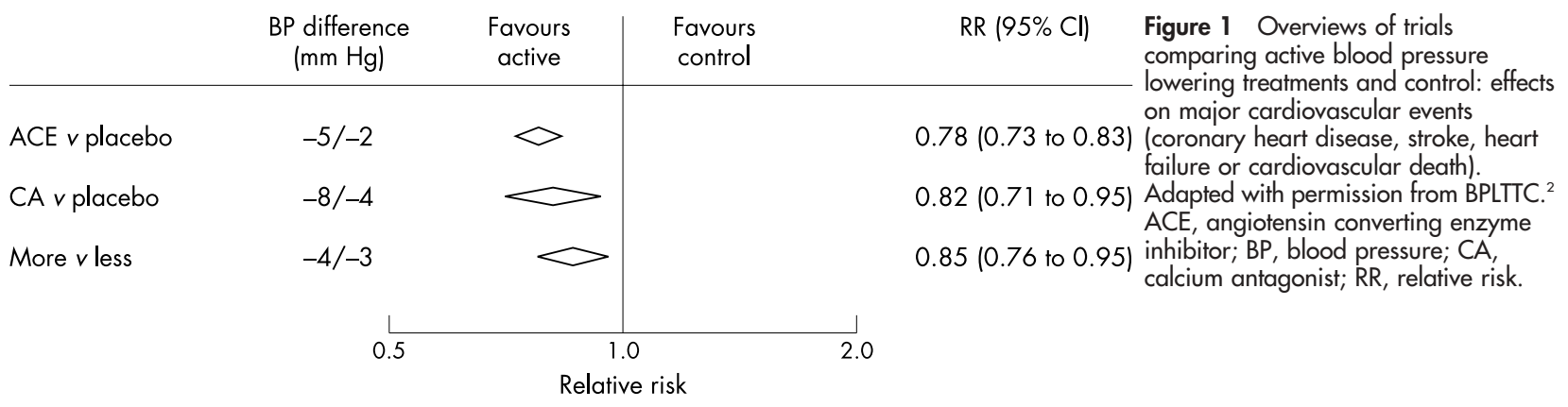

analysis combined data from LIFE, SCOPE, and two trials conducted among diabetic populations (the irbesartan diabetic nephropathy trial (IDNT), and the reduction of endpoints in NIDDM with the angiotensin II antagonist losartan (RENAAL) study). Although interpretation of the combined data is difficult because of the different trial designs and study populations, ARBs appear to reduce the risks of stroke, heart failure, and combined major cardiovascular events. It remains unclear, however, how much of this benefit is attributable to the greater blood pressure lowering (mean $2 / 1 \mathrm{~mm} \mathrm{Hg}$ ) achieved with the ARB based regimens. On the basis of current evidence, therefore, it appears that angiotensin receptor blockers are effective blood pressure lowering treatments and that they reduce the risk of vascular events. However, whether they have genuine advantages, beyond blood pressure lowering, over other drug classes remains to be confirmed by further clinical trials.

\section{TRIALS COMPARING DIFFERENT BLOOD PRESSURE LOWERING DRUGS}

The antihypertensive and lipid-lowering treatment to prevent heart attack trial (ALLHAT) ${ }^{5}$ is the biggest trial of blood pressure lowering treatment to date. It involved 33357 participants, aged 55 years or older, with hypertension and at least one other CHD risk factor. Patients were randomised to receive a diuretic (chlorthalidone), calcium channel blocker (amlodipine), or ACE inhibitor (lisinopril), and followed up for an average of 4.9 years. There were small but significant differences in achieved blood pressure. For the primary end point of fatal or non-fatal CHD, there were no differences between the three drugs. There were, however, modest differences between the treatments for other end pointsfor example, lisinopril was associated with a $10 \%$ greater risk of combined cardiovascular events compared with chlorthalidone, a result which is likely to be at least partly explained by the blood pressure differences achieved.

Further data come from the recent BPLTTC overviews of trials that compared blood pressure lowering drugs of different classes (fig 2). ${ }^{2}$ These showed that for major cardiovascular events there are no differences between the effects of conventional blood pressure lowering regimens (based on diuretics or $\beta$ blockers) and those based on ACE inhibitors or calcium channel blockers. The confidence intervals around these combined estimates of effect are narrow, suggesting that even if true differences do exist, they are likely to be small. In view of this, the choice of first line blood pressure lowering drug is likely to be of relatively little importance, particularly since the majority of patients will need at least two agents to achieve blood pressure targets.

An important final point to note is that in "high risk" individuals, such as those with diabetes, CHD, cerebrovascular or other vascular disease, there is now good evidence that lowering blood pressure is beneficial even among individuals with blood pressures of $140 / 90 \mathrm{~mm} \mathrm{Hg}$ or less. ${ }^{6-8}$

\section{HYPERLIPIDAEMIA}

As with blood pressure, there are strong and continuous associations between cholesterol and the risks of CHD and non-haemorrhagic stroke. ${ }^{10}$ This paper will consider evidence of the benefits of lipid lowering in the light of the results of four major studies published in the last two years. ${ }^{11-14}$

The largest of these, the heart protection study (HPS), ${ }^{11}$ included 20536 participants, aged 40-80 years, with total cholesterol $\geqslant 3.5 \mathrm{mmol} / \mathrm{l}$. Participants were "high risk" patients with existing CHD, other occlusive vascular disease or diabetes. They were randomised to simvastatin $40 \mathrm{mg}$ or placebo, and followed up for five years. The average difference in low density lipoprotein (LDL) cholesterol concentration achieved between the groups was $1 \mathrm{mmol} / \mathrm{l}$ (29\%). Active treatment was associated with a $24 \%$ relative reduction in the risk of the composite end point of major vascular events $(p<0.0001)$. All cause mortality, the trial's primary end point, was reduced in the simvastatin group by $13 \%(\mathrm{p}=0.0003)$, largely due to a highly significant $17 \%$ reduction in the relative risk of vascular death $(7.6 \% v 9.1 \%$, $\mathrm{p}<0.0001)$. There was no increase in the risk of nonvascular death in the simvastatin group. This is an important finding since there has been some concern that lipid lowering drugs may increase the risk of cancer. Also, as predicted from the observational data, ${ }^{10}$ there was a $25 \%$ reduction in the relative risk of stroke $(4.3 \% \vee 5.7 \%, \mathrm{p}<0.0001)$.

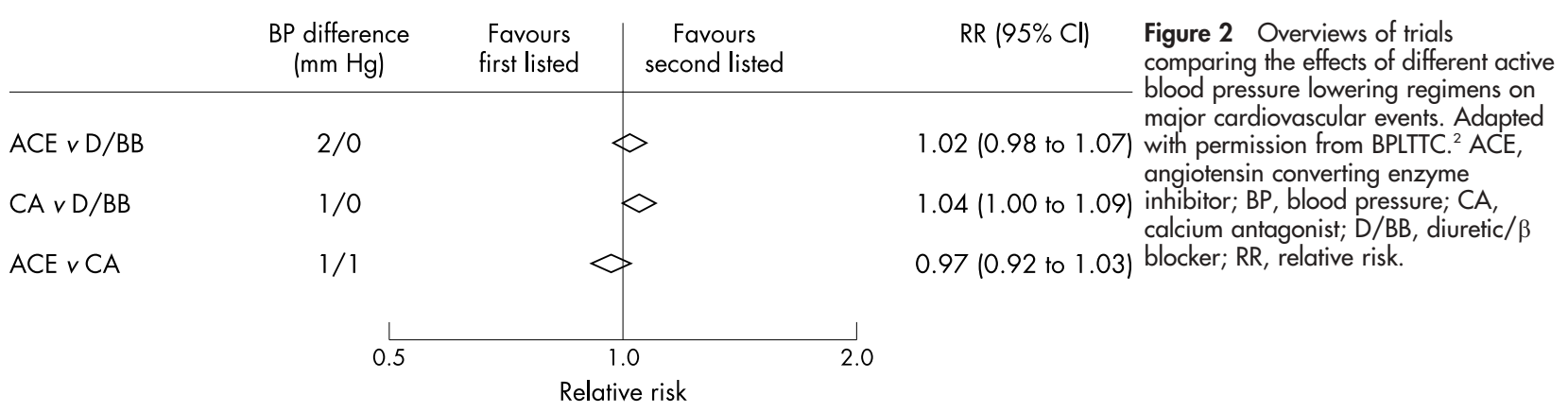


The pravastatin in elderly individuals at risk of vascular disease (PROSPER) study ${ }^{12}$ involved 5804 elderly patients (aged 70-82) with a history of, or risk factors for, vascular disease, and cholesterol concentrations of $4.0-9.0 \mathrm{mmol} / \mathrm{l}$. Patients were randomised to pravastatin $40 \mathrm{mg}$ or placebo, and followed up for an average of 3.2 years. Active treatment reduced LDL cholesterol by $34 \%$ and led to a $15 \%$ relative reduction $(p=0.014)$ in the primary end point of major vascular events (a composite of coronary death, non-fatal myocardial infarction, and fatal and non-fatal stroke).

The Anglo-Scandinavian cardiac outcomes trial-lipid lowering arm (ASCOT-LLA) ${ }^{13}$ included 10305 participants with hypertension and at least three other cardiovascular risk factors, and a total cholesterol concentration of $\leqslant 6.5 \mathrm{mmol} /$ l. (It was felt to be unethical to randomise those with higher cholesterol values.) Patients were randomised to atorvastatin $10 \mathrm{mg}$ or placebo. The study was terminated prematurely after a median follow up period of 3.3 years, when the data safety monitoring board decided that there was clear evidence of benefit of atorvastatin. Active treatment achieved relative reductions in total and LDL cholesterol of $24 \%$ and $35 \%$, respectively. This was associated with a $36 \%$ relative reduction in the primary end point of fatal and non-fatal CHD $(p=0.0005)$ and a $27 \%$ reduction in the risk of stroke $(\mathrm{p}=0.02)$.

The lipid lowering component of ALLHAT-LLT ${ }^{14}$ involved 10355 participants, aged 55 or older, who had hypertension, at least one other CHD risk factor, and LDL cholesterol concentrations of $3.1-4.9 \mathrm{mmol} / \mathrm{l}$ (or lower if patients had CHD). Patients were randomised to open label pravastatin $40 \mathrm{mg}$ or "usual care" because it was felt unethical to randomise to placebo. After a mean follow up of 4.8 years, there was no significant difference in the primary end point (all cause mortality), or in the rate of fatal and non-fatal CHD. Caution is needed in interpreting this negative result. In the event, about one third of the "usual care" group commenced lipid lowering treatment during the trial and, as a result, only modest differences in total and LDL cholesterol (10\% and 17\%, respectively) were achieved between the active and placebo groups. This failure to achieve good separation in cholesterol values between the groups is likely to explain the lack of benefit of pravastatin observed in the trial. This was illustrated in the paper reporting the ALLHATLLT results: when the result is plotted against the achieved reduction in total cholesterol, it remains consistent with what would be predicted for the level of lipid lowering achieved (fig 3).

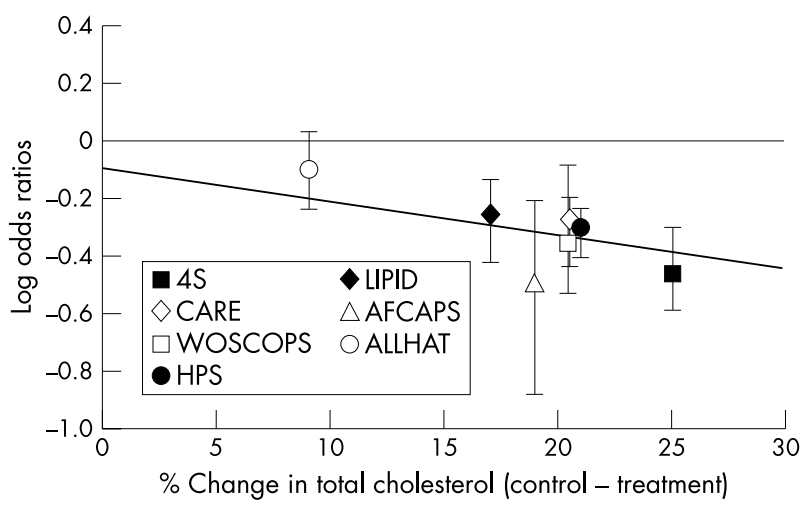

Figure 3 Reductions in coronary heart disease event rates according to change in total cholesterol; regression line from meta-analysis of 45 trials of lipid lowering published before 31 December 2000. Adapted with permission from ALLHAT-LLT. ${ }^{14}$

\section{CONCLUSION}

In hypertension, trials have now shown clear evidence of benefits of blood pressure lowering with all commonly used classes of drug. More intensive blood pressure lowering has also been shown to be beneficial. While there is some evidence of differences between the effects of different blood pressure lowering drugs on cause specific outcomes, such as myocardial infarction or stroke, no consistent differences have been found in the effects of different classes of drug on combined major cardiovascular events.

There is also now good evidence that cholesterol lowering with statins reduces the risks of death and cardiovascular events in "high risk" patients, even at concentrations of total cholesterol as low as $3.5 \mathrm{mmol} / \mathrm{l}$. These benefits are consistent across a range of patient subgroups and appear to be proportional to the magnitude of cholesterol lowering achieved.

\section{REFERENCES}

1 Collins R, MacMahon S. Blood pressure, antihypertensive drug treatment and the risks of stroke and of coronary heart disease. $\mathrm{Br}$ Med Bull 1994:50:272-98.

2 Blood Pressure Lowering Treatment Trialists' Collaboration. Effects of different blood-pressure-lowering regiments on major cardiovascular events: results of prospectively-designed overviews of randomised trials. Lancet 2003;362:1527-35.

3 Dahlof B, Devereux RB, Kjeldsen SE, et al for the LIFE study group. Cardiovascular morbidity and mortality in the losartan intervention for endpoint reduction in hypertension study (LIFE): a randomised trial against atenolol. Lancet 2002;359:995-1003.

4 Lithell H, Hansson L, Skoog I, et al for the SCOPE study group. The study on cognition and prognosis in the elderly (SCOPE): principal results of a randomised double-blind intervention trial. J Hypertens 2003;21:875-86.

5 The ALLHAT Officers and Co-ordinators for the ALLHAT Collaborative Research Group. Major outcomes in high-risk hypertensive patients randomised to angiotensin-converting enzyme inhibitor or calcium channel blocker vs diuretic. The antihypertensive and lipid-lowering treatment to prevent heart attack trial (ALLHAT). JAMA 2002;288:2981-97.

6 The Heart Outcomes Prevention Evaluation Study Investigators. Effects of an angiotensin-converting-enzyme inhibitor, ramipril, on cardiovascular events in high-risk patients. N Engl J Med 2000;342:145-53.

7 PROGRESS Collaborative Group. Randomised trial of a perindopril-based blood-pressure-lowering regimen among 6105 individuals with previous stroke or transient ischaemic attack. Lancet 2001;358:1033-41.

8 EUROPA Investigators. Efficacy of perindopril in reduction of cardiovascular events among patients with stable coronary artery disease: randomised, double-blind, placebo-controlled, multicentre trial (the EUROPA study). Lancet 2003;362:782-8.

9 Eastern Stroke and Coronary Heart Disease Collaborative Research Group. Blood pressure, cholesterol and stroke in eastern Asia. Lancet 1998;352:1801-7.

10 Zhang X, Patel A, Horibe H, et al for the Asia Pacific Cohort Studies Collaboration. Cholesterol, coronary heart disease, and stroke in the Asia Pacific region. Int J Epidemiol 2003;32:563-72.

11 Heart Protection Study Collaborative Group. MRC/BHF heart protection study of cholesterol lowering with simvastatin in 20536 high-risk individuals: a randomised placebo-controlled trial. Lancet 2002;360:7-22.

12 Shepherd J, Blauw GJ, Murphy MB, et al on behalf of the PROSPER study group. Pravastatin in elderly individuals at risk of vascular disease (PROSPER): a randomised controlled trial. Lancet 2002;360:1623-30.

13 Sever PS, Dahlof B, Poulter NR, et al for the ASCOT investigators. Prevention of coronary and stroke events with atorvastatin in hypertensive patients who have average or lower-than-average cholesterol concentrations, in the AngloScandinavian cardiac outcomes trial-lipid lowering arm (ASCOT-LLA): a multicentre randomised controlled trial. Lancet 2003;361:1149-58.

14 The ALLHAT Officers and Co-ordinators for the ALLHAT Collaborative Research Group. Major outcomes in moderately hypercholesterolemic, hypertensive patients randomised to pravastatin vs usual care. The antihypertensive and lipid-lowering treatment to prevent heart attack trial (ALLHAT-LLT). JAMA 2002;288:2998-3007.

\section{DISCUSSION}

Question: It is being suggested to GPs that treating hypertensive patients with lipid lowering agents will help to reduce mortality. This is a distortion of the results of the ASCOT trial, in which patients had three or more major other risk factors. We know that within our hypertensive patients some are patients with metabolic syndrome and these almost certainly will be helped by statins, but a significant number of patients may not be helped by statins. 
Dr Chapman: It is not the case that all hypertensive patients have been shown to benefit. In ASCOT, patients had to have hypertension and additional risk factors. Based on the evidence at the moment, it is such high risk patients who have been shown to benefit. You might want to extrapolate those results to all hypertensive patients, but the evidence is not currently available.

Question: There is no argument for any threshold for treatment, in that age and gender are the major determinants of risk, so should we not accept the polypill argument and treat everybody?

Dr Chapman: I would say that in high risk people-such as those with vascular disease-the initial cholesterol level should not be of importance. But there must be a level of absolute risk at which the risks of statins, aspirin or whatever, outweigh the benefits. The polypill principle is an interesting one and there has been a lot of debate about it since the concept was published in the $B M J$. But clinical trials are needed before it can be taken as a serious proposition.
Question: I am concerned about the fact that in the ALLHAT trial there was an increased rate of diabetes in the chlorthalidone arm. When we start patients on antihypertensives aged 50 we are going to have them on treatment for 20,30 , or maybe even 40 years. Are we going to be creating diabetes in these patients, particularly the ones who have the metabolic syndrome?

Dr Chapman: That potentially is a problem. Both diuretics and $\beta$ blockers can worsen a person's metabolic profile. Most patients are going to need at least two tablets to control their blood pressure. I believe that in most cases one of those should be a diuretic because they are so effective. Yes, you may get an increase in the number of people who develop diabetes. But I don't think that should prevent us from treating the blood pressure effectively. We need to monitor glucose levels, we need to get people to modify their lifestyle accordingly, we need to identify and treat those who develop diabetes, but it shouldn't affect the choice of treatment. 